\title{
BMJ Open Sarcome-13/OS2016 trial protocol: a multicentre, randomised, open-label, phase II trial of mifamurtide combined with postoperative chemotherapy for patients with newly diagnosed high- risk osteosarcoma
}

Caroline Brard, ${ }^{1,2}$ Sophie Piperno-Neumann, ${ }^{3}$ Jessy Delaye, ${ }^{4}$ Laurence Brugières, ${ }^{5}$ Lisa V Hampson, ${ }^{6,7}$ Gwénaël Le Teuff, ${ }^{1,2}$ Marie-Cécile Le Deley, ${ }^{1,8}$ Nathalie Gaspar ${ }^{5}$

To cite: Brard C, PipernoNeumann S, Delaye J, et al. Sarcome-13/0S2016 trial protocol: a multicentre, randomised, open-label, phase II trial of mifamurtide combined with postoperative chemotherapy for patients with newly diagnosed highrisk osteosarcoma. BMJ Open 2019;9:e025877. doi:10.1136/ bmjopen-2018-025877

- Prepublication history and additional material for this paper are available online. To view these files, please visit the journal online (http://dx.doi. org/10.1136/bmjopen-2018025877).

Received 6 August 2018 Revised 13 March 2019 Accepted 4 April 2019

Check for updates

(C) Author(s) (or their employer(s)) 2019. Re-use permitted under CC BY-NC. No commercial re-use. See rights and permissions. Published by BMJ.

For numbered affiliations see end of article.

Correspondence to Dr Marie-Cécile Le Deley; m-ledeley@0-lambret.fr

\section{ABSTRACT}

Introduction The controversial results on the mifamurtide efficacy associated with chemotherapy, issued from the American INT-0133-study, in localised osteosarcomas, and the underpowered analysis performed separately in metastatic patients, should be clarified to homogenise international use of this promising drug. The European Commission has granted a marketing authorisation to mifamurtide combined with postoperative chemotherapy in localised osteosarcomas but not in metastatic patients, while the Food and Drug Administration (FDA) has denied this authorisation.

Methods and analysis Sarcome-13/0S2016 trial is a multicentre randomised open-label phase II trial evaluating the survival benefit of mifamurtide administered during 36 weeks in combination with postoperative chemotherapy versus chemotherapy alone, in patients $>2$ and $\leq 50$ years with newly diagnosed high-risk localised or metastatic osteosarcoma. The main objective is to evaluate the impact on event-free survival (EFS) of mifamurtide on intention-totreat population. The secondary objectives are to evaluate the impact of mifamurtide on overall survival, to evaluate the feasibility and toxicity of the planned treatment, to correlate biology/immunology with the mifamurtide efficacy/toxicity. With a total of 126 enrolled patients and 51 events, the power is $80 \%$ if mifamurtide is associated with an 18\% improvement of the 3-year EFS (52\%vs $70 \%$, equivalent to an $\mathrm{HR}=0.55$ ), with a one-sided logrank test alpha $=10 \%$. As relevant historical data are available (aggregate treatment effect from the INT-0133 trial and individual data from the control group of the Sarcome-09/ OS2006 trial), a Bayesian analysis is also planned.

Ethics and dissemination This study was approved by the 'Comité de Protection des Personnes lle de France l' (12/06/2018), complies with the Declaration of Helsinki and French laws and regulations, and follows the International Conference on Harmonisation E6 Guideline for Good Clinical Practice. The trial results, even if they are inconclusive, as well as biological ancillary studies will be presented at appropriate international congresses and published in international peer-review journals.

\section{Strengths and limitations of this study}

- Multicentre, randomised, phase II trial design to evaluate the efficacy of mifamurtide combined with postoperative chemotherapy, in children and adults with high-risk osteosarcoma.

- By using ifosfamide-based chemotherapy for all patients, we avoid the possible issue in interpreting the data due to an interaction between mifamurtide and chemotherapy regimen (with or without ifosfamide) as published in the original article of Meyers et al. (J Clin Oncol).

- The relatively small sample size may limit the findings, but it fits with the rare disease setting and a realistic time frame.

- Incorporating historical data (individual control data and aggregate treatment effect) using a power and mixture prior for a Bayesian survival augments the design and analysis of the Sarcome-13/0S2016 trial.

Trial registration number EudraCT 2017-001165-24, NCT03643133

\section{INTRODUCTION}

Epidemiology and prognosis of osteosarcoma

Osteosarcoma is the most common primary malignant bone tumour with a peak incidence in adolescents and young adults (AYA). However, in France, this rare disease represents less than $10 \%$ of all AYA cancers with 150 new cases/year, including adults. Osteosarcoma treatment relies on preoperative chemotherapy, tumour surgical resection and postoperative chemotherapy. With this multidisciplinary approach, the 3-year event-free survival (EFS) rate reaches $70 \%$ in patients with non-metastatic osteosarcoma. 
In France, the chemotherapy regimen used for treatment depends on age: children/adolescents are treated with the combination of methotrexate, etoposide and ifosfamide (M-EI), ${ }^{1}$ while adults receive a combination of doxorubicin, cisplatin and ifosfamide (API-AI) ${ }^{2}$ These regimens formed the backbone preoperative chemotherapy of the recent Sarcome-09/OS2006 trial. ${ }^{3}$ Postoperative chemotherapy is adapted to the metastatic status at diagnosis and, for patients with a localised disease, to the histological response to neoadjuvant chemotherapy according to Huvos' grading.

The main risk factors of relapse are the presence of metastases at diagnosis, a non-operable disease and poor histological response to preoperative chemotherapy, usually defined by $\geq 10 \%$ residual tumour cells. In the Sarcome-09/OS2006 trial, patients with skip metastasis had a poorer outcome than patients with localised disease, close to patients with metastatic disease, and we considered them as metastatic even if the skip metastasis is the unique metastatic lesion. The 3-year EFS in these high-risk patients is around $40 \%$, which has not improved in the last 20 years despite several clinical trials. ${ }^{34}$ To address this issue, new treatment strategies for patients with high-risk osteosarcoma are needed.

\section{Mifamurtide (L-MTP-PE, MEPACT) in osteosarcoma}

Mifamurtide is a fully synthetic lipophilic derivative of the muramyl dipeptide (MDP), encapsulated into liposomes. It binds to extracellular Toll-like receptor-4, activating monocytes and macrophages, and promotes antitumour activity. However, mifamurtide also activates antitumour function of macrophages by intracellular nucleotide-binding oligomerisation domain-2 (NOD) receptor. Mifamurtide enters into macrophages, is degraded into MDP and binds to NOD-2 receptors. It induces NF- $\mathrm{KB}$ release through activation of receptor-interacting serine/ threonine protein kinases (RICK) signaling pathway and secretion of inflammatory cytokines such as TNFa, IL-6, IL-8 and IL-1. ${ }^{5}$ Preclinical studies showed mifamurtide has similar immunostimulatory effects on these cells as the natural MDP, with the additional advantage of a longer half-life in plasma, lower toxicity and better efficacy. The activation of the monocytes/macrophages and dendritic cells may also activate other innate immune cells such as natural killer cells, and may generate an adaptive immune response by $\mathrm{T}$ cells. Human monocytes/macrophages, after in vitro activation with mifamurtide, specifically recognised tumour cells and were not cytotoxic to normal cells. ${ }^{7}$ In vivo administration of mifamurtide resulted in the inhibition of tumour growth in mouse and rat models of lung metastasis, skin and hepatic cancer, and fibrosarcoma ${ }^{89}$ Interest in mifamurtide for the treatment of osteosarcoma arises from the remarkable results achieved in dogs, in which spontaneous osteosarcoma is common, and similar to the human disease. Randomised studies in dogs of either mifamurtide alone, or in combination with chemotherapy, showed a significantly improved survival in dogs treated with mifamurtide. ${ }^{10} 11$ Results in animal tumour models do not support a significant interaction of most chemotherapeutic drugs on macrophage activation by mifamurtide. $^{12}$

\section{Trial rationale}

From 1993 to 1997, the randomised, phase III INT-0133 trial, considering patients with newly diagnosed osteosarcoma, and younger than 30 , addressed two questions in a factorial design: efficacy of ifosfamide in addition to MAP chemotherapy (methotrexate-doxorubicincisplatin); and efficacy of mifamurtide in addition to postoperative chemotherapy. In the 662 patients enrolled with a localised resectable osteosarcoma, the first analysis showed mifamurtide might improve EFS, but a potential interaction between ifosfamide and mifamurtide hampered the results interpretation. ${ }^{13}$ A second publication on the same population with a longer follow-up reported a significant benefit of mifamurtide on overall survival (OS) (HR 0.71, 95\% CI0.52 to $0.96, \mathrm{p}=0.03$ ) and a 6-year survival improvement rate from $70 \%$ to $78 \%$, with no significant ifosfamide/mifamurtide interaction. ${ }^{14}$ In addition, 91 patients with synchronous metastatic osteosarcoma recruited in this trial were analysed separately: mifamurtide effect size on OS was similar in this group (HR $0.72,95 \%$ CI 0.40 to 1.30 ), but not significant $(\mathrm{p}=0.27){ }^{15}$

A retrospective study on metastatic/relapsed osteosarcoma confirmed mifamurtide safety. ${ }^{16}$ The most frequent side effects of mifamurtide are chills, fever, fatigue, nausea, tachycardia and headache with mild to moderate grade. Mifamurtide combined with nephrotoxic (cisplatin, ifosfamide) and hepatotoxic (high-dose methotrexate) chemotherapies in the INT-0133 trial did not increase these toxicities. ${ }^{13-15}$

Based on these results, the European Medicines Agency granted a centralised marketing authorisation on $6 \mathrm{March}$ 2009 for mifamurtide combined with postoperative chemotherapy for patients between 2 and 30 years old and presenting a high-grade non-metastatic osteosarcoma with complete macroscopic resection (EU/1/08/502/001). However, in the USA, no approval has been obtained. In France, the French Transparency Commission required additional investigation before considering mifamurtide as a standard in front-line therapy of osteosarcoma. In several European countries, mifamurtide use is still limited as the results of the INT-0133 trial have been a matter of debate between key-opinion leaders. ${ }^{17}$ In addition, European Union authorisation does not include patients over 30 , as well as patients with primary metastatic disease for whom outcome improvement is still challenging.

\section{Aim of the study}

In this context, further investigations of first-line treatment with mifamurtide in osteosarcoma are necessary, especially for patients with a high risk of relapse (metastases at diagnosis or poor histological response to neoadjuvant chemotherapy). As the current postoperative chemotherapy regimens used for the treatment of osteosarcoma 
in France contain ifosfamide, the possible interaction with mifamurtide would not jeopardise the evaluation of the benefit associated with mifamurtide. Sarcome-13/ OS2016 trial intends to evaluate the survival benefit of mifamurtide combined with postoperative chemotherapy for treating patients with high-risk osteosarcoma.

EFS will be used as a primary endpoint. We will also study the impact of mifamurtide on OS, and the feasibility of the planned treatment with calculation of cumulative dose and dose intensity of mifamurtide and chemotherapy. Acute and long-term toxicity will be evaluated, as well as biomarkers that could be surrogate markers of mifamurtide pharmacological effect, or predictive factors of efficacy and/or toxicity of mifamurtide. If the efficacy of mifamurtide is confirmed, this may allow the use of a currently promising, original drug, in patients with osteosarcoma with the highest risk of failure.

This article reports the Sarcome-13/OS2016 trial protocol V.2.0 of the 28 May 2018.

\section{METHODS AND ANALYSIS \\ Study design}

Sarcome-13/OS2016 is a French, multicentre, randomised, open-label, phase II trial, with two parallel groups in first-line treatment of high-risk patients with osteosarcoma. This trial is part of a study recruiting all patients aged 2-50years old with a newly diagnosed high-grade osteosarcoma. Enrolment in the randomised trial will take place after neoadjuvant chemotherapy and surgery. After check of eligibility criteria for the randomised part of the trial, and after informed written consent is signed, the investigator will proceed with the randomisation through an online electronic case report form (e-CRF). A password non-disclosure certificate is signed by the principal investigator engaging his responsibility regarding the confidentiality of the access codes for all users of the e-CRF in his centre. Participation to biological ancillary studies is optional, and subject to a separate consent form. The biological samples will be shipped and centralised at different 'Centre des Ressources Biologiques' depending on the samples, under the responsibility of a designated person. Postoperative treatment will be allocated by randomisation (1:1) between: arm-A (control arm: postoperative chemotherapy alone) and arm-B (experimental arm: postoperative chemotherapy + mifamurtide). A dynamic allocation process (minimisation) will be used to obtain a balanced distribution of treatment groups according to planned postoperative chemotherapy aged-based regimens and risk groups: (1) localised disease at diagnosis and poor histological response, (2) metastatic disease at diagnosis (skip or distant metastases) and complete removal of lesions before randomisation and good histological response of the primary tumour, (3) metastatic disease at diagnosis (skip or distant metastases) and planned complete removal of lesions, and good histological response of the primary tumour, (4) metastatic disease at diagnosis and complete removal of lesions before randomisation and poor histological response of the primary tumour, (5) metastatic disease at diagnosis and planned complete removal of lesions and poor histological response of the primary tumour.

Since osteosarcoma is a rare disease, a large phase III trial with standard alpha level cannot be performed within a reasonable time frame. A randomised phase II trial evaluating the efficacy of mifamurtide at a relaxed alpha level in high-risk patients will produce new independent data, which may be combined with results from the previous INT-0133 trial.

\section{Patient selection criteria}

Registration criteria at diagnosis

The following criteria must be met at diagnosis for registration in the study:

1. All newly diagnosed, biopsy-proven, high-grade osteosarcoma, whatever the initial extension of the disease.

2. Age 2-50.

3. Normal haematological, renal, cardiac and hepatic functions.

4. Planned neoadjuvant chemotherapy as follows:

a. M-EI regimen for patients $\leq 25$ years old.

b. API-AI regimen for patients 26-50years old.

5. Written informed consent from patients and/or their parents/guardians before enrolment and any study-related procedure.

6. Affiliation to a social insurance regimen.

\section{Inclusion criteria for randomisation}

Patients must meet the following criteria:

1. Histologically proven, confirmed by expert pathologists panel, high-grade osteosarcoma.

2. Registered at diagnosis into the study.

3. Primary tumour resected after preoperative chemotherapy.

4. Osteosarcoma classified as high risk because of at least one risk factor:

a. Presence of distant metastases or skip metastases at diagnosis.

b. Poor histological response to preoperative chemotherapy ( $>10 \%$ residual viable cells on the analysis of the primary tumour surgical specimen).

5. Preoperative chemotherapy combining

a. M-EI regimen for patients $\leq 25$ years old.

b. API-AI regimen for patients 26-50years old.

6. Screening laboratory values must meet the following criteria (using Common Terminology Criteria for Adverse Events [CTCAE], V.5) and should be obtained within 7 days prior to randomisation:

a. Absolute neutrophil count $\geq 1 \times 10^{9} / \mathrm{L}$.

b. Platelets $\geq 100 \times 10^{9} / \mathrm{L}$.

c. Haemoglobin $\geq 8 \mathrm{~g} / \mathrm{dL}$.

d. Alanine aminotransferase (ALT) and Aspartate aminotransferase (AST) $\leq 2.5 \times$ XLN in the absence of liver metastases or $\leq 5 \times \mathrm{xULN}$ in the presence of liver metastases. 
e. Total bilirubin $\leq 2 \mathrm{x}$ ULN (except Gilbert syndrome: $<3.0 \mathrm{mg} / \mathrm{dL}$ ) or total bilirubin $\leq 5.0 \times \mathrm{xLN}$ in the presence of liver metastases.

f. Creatinine clearance $\geq 60 \mathrm{~mL} / \mathrm{min} / 1.73 \mathrm{~m}^{2}$ according to the Schwartz or Cockroft formula according to patient's age.

7. Women of childbearing potential must have a negative serum or urine pregnancy test done within 7 days prior to randomisation.

8. Provision of dated and signed written informed consent for the randomised trial prior to any study-specific procedures, sampling and analyses.

9. Patient fit to undergo protocol treatment and follow-up.

\section{Non-inclusion criteria for randomisation}

Patients with any of the following conditions are not included in the study:

1. Low-grade osteosarcoma, parosteal or periosteal osteosarcoma.

2. Prior history of other malignancies other than study disease unless the patient has been free of the disease for at least 3 years.

3. Osteosarcoma with multiple metastases for whom complete removal is not expected to be feasible even after shrinkage with chemotherapy.

4. Progressive disease at any site during initial chemotherapy, confirmed before randomisation time and not totally resected during surgery.

5. Any medical condition precluding treatment with protocol chemotherapy.

6. Fractional Shortening $<28 \%$ or Left Ventricular Ejection Fraction (LVEF) $<50 \%$ before treatment (only for API postoperative chemotherapy) by echocardiogram or multiple-gated acquisition (MUGA) scan.

7. Pregnancy or breast feeding

8. Hypersensitivity to the active substance or to any of the excipients.

9. Concurrent use of immunodepressive treatment such as cyclosporine, tacrolimus or other calcineurin inhibitors.

10. Concurrent use with high-dose non-steroidal anti-inflammatory drugs.

11. Inflammatory or autoimmune disease, allergy or asthma requiring a chronic use of steroid treatment that cannot be stopped.

12. Patients with positive test for HIV or known AIDS.

13. Patients with positive tests for hepatitis-B virus surface antigen or hepatitis-C virus RNA indicating active or chronic infection.

\section{Study description}

\section{Intervention}

After preoperative chemotherapy and surgery of the primary tumour, patients presenting high-risk osteosarcoma will be randomised to receive the treatment allocated, either:

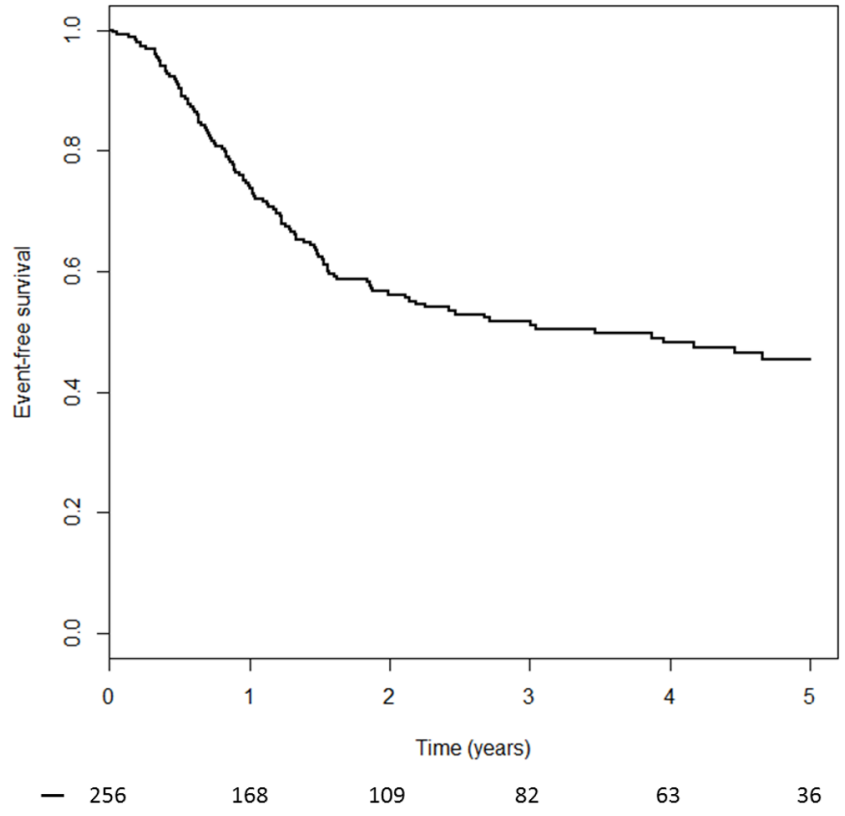

Figure 1 Treatment scheme according to age.

- Arm-A: Postoperative chemotherapy alone, according to age group.

- Arm-B: Postoperative chemotherapy, according to age group + mifamurtide.

Postoperative chemotherapy is part of standard of care for high-risk osteosarcoma. Chemotherapy should be administered as per local practice at each investigator site. The choice of the chemotherapy regimen is based on experience from Sarcome-09/OS2006 trial, and treatments are determined according to patient's age (figure 1):

- Patients $\leq 25$ years old, (M)-API regimen: one course of high-dose methotrexate followed by five API courses, every 21 days.

- Patients 26-50years old, EI-regimen: five EI courses, every 21 days.

Postoperative treatment will have to start as soon as possible after randomisation and within a maximum of 7 days postrandomisation.

Patients allocated to the experimental arm will receive mifamurtide $2 \mathrm{mg} / \mathrm{m}^{2}$ intravenously two times weekly for 12 weeks, at least 3 days apart, then weekly for 24 weeks, starting at the same time of postoperative chemotherapy.

Surgery of the metastatic sites will be performed 1-2 weeks after the surgery of the primary site and before randomisation, if possible. If a surgery of both lungs is indicated, one side should be operated before randomisation while the second side will be operated 6 weeks after the start of postoperative treatment. This will allow a sequential biological/immune study in the experimental arm. If the disease burden remains to be operated, the second surgery can be delayed. In that case, an optional biopsy will be proposed to the patients included in the experimental arm. During the postoperative chemotherapy period, patients will have a physical examination 
and biological tests before each treatment course, every 3 weeks. Radiological, and echocardiography or cardiac MUGA scintigraphy, and audiogram will also be performed. During the mifamurtide period without chemotherapy, patients will be seen in clinic every week by the physician (before each mifamurtide infusion) and will have standard clinical assessment. They will also have a formal visit every 3 months. Follow-up assessment of the disease will be performed (from last treatment administration) every 3 months for 3 years, then every 6 months for 2 years, then every year up to 10 years for all patients. Follow-up assessment of late treatment effects will be performed 1 and 3 years after the end of postoperative chemotherapy.

All medications for the treatment of symptoms are authorised, and their type, posology and duration of administration will be recorded. Patients should receive full supportive care during the study, including transfusions and analgesics, as appropriate.

As mifamurtide has a lipophilic formulation, it is recommended to separate the times of administration of mifamurtide and doxorubicin, or other lipophilic drugs.

Prohibited concomitant treatments are:

- Immunodepressive treatment.

- High-dose non-steroidal anti-inflammatory drugs.

- Chronic use of steroid treatment.

- Systematic use of steroids as antiemetic prophylaxis.

Premature treatment discontinuation may be due to the following reasons: disease progression, unacceptable toxicity, intercurrent conditions that preclude the continuation of treatment, patient choice or physician decision. Except in case of consent withdrawal for the participation in the study, patient follow-up will continue in compliance with the protocol and follow-up data will be collected until the end of the trial.

\section{Outcome measures \\ Study objectives}

The primary objective of the trial is to evaluate the survival benefit (EFS) of mifamurtide administered during 36 weeks combined with postoperative chemotherapy compared with postoperative chemotherapy alone. The secondary objectives are to evaluate: (1) the impact on OS of mifamurtide, (2) the feasibility and safety of mifamurtide administration during and after postoperative chemotherapy, (3) the mifamurtide effect on antitumour immunity in patients with sequential surgery of lung metastases, (4) biomarkers that could be surrogate of mifamurtide pharmacological effect or predictive factors of efficacy and/or toxicity of mifamurtide, (5) the tumour microenvironment in osteosarcoma and its correlation with clinical characteristics and outcome and (v6) the potential new therapeutic targets for future combinations (Whole Exon Sequencing, RNAseq, Immuni-Histo-Chemistry, flow cytometry, ELISA).

\section{Measurement tools}

EFS is defined from the randomisation date to the time of first event (locoregional or distant relapse or progression, second malignancy, death from any cause). Observations will be censored at the date of last follow-up visit for the patients remaining in first complete remission. OS is defined as the time between the randomisation date and the date of death, whatever the cause of death.

Treatment safety evaluation will be based on adverse event (AE) occurrence, the use of concomitant treatments and treatment modifications. AEs will be graded according to the toxicity criteria (NCI-CTCAE) V.5.0.

The software Clinsight will be used for data entry, management and archiving data.

\section{Statistical considerations}

\section{Sample size}

Due to the rare disease setting, we relaxed the alpha level of the one-sided log-rank test to $10 \%$, and a pragmatic recruitment target has been set of accruing 126 patients over 3 years. If this target is met, the power is $80 \%$ if the true HR=0.55 (18\% improvement of the 3-year EFS, $52 \%$ vs $70 \%$ ), which requires 51 events if the analysis is performed at the end of study. The minimum follow-up will be 2 years from randomisation, leading to a total duration of the trial of 5 years. A long-term follow-up is also planned beyond the primary analysis, up to 10 years from randomisation. The sample size was computed using EAST (EAST V.6.4, Cytel) assuming a piecewise exponential survival model with two knots at 0.3 and 1.6 years, consistent with the observed 1-year $\mathrm{EFS}=74 \%$, 2-year $\mathrm{EFS}=56 \%$, 3-year $\mathrm{EFS}=52 \%$ and 5 -year $\mathrm{EFS}=45 \%$ in high-risk patients from Sarcome-09/OS2006 trial (hazard rates: $\lambda_{1}=0.1065, \lambda_{2}=0.3723$ and $\left.\lambda_{3}=0.0878\right)$.

In the previous Sarcome-09/OS2006 trial, the proportion of patients lost to follow-up was $<1 \%$ at 3 years. This has a very limited impact on the sample size calculation; it only requests a slightly longer follow-up duration from 2.048 to 2.091 for the last accrued patient. Based on Sarcome-09/OS2006 data, the estimated proportion of patients fulfilling eligibility criteria for the randomised trial is $43 \%$ among all patients enrolled in the study. As we expect an attrition rate of $25 \%$ (refusal of participation in the randomised trial), we estimate that $31.5 \%$ of the patients enrolled at diagnosis will be enrolled in the randomised trial. Consequently, 390 registered patients $(126 /(0.43 * 0.75)=390)$ may be required for the study to reach the 126 patients and 51 events for the randomised trial.

\section{Statistical analysis}

EFS curves will be estimated using the Kaplan-Meier method. Difference between EFS curves will be tested using a log-rank test at a one-sided alpha $=10 \%$. As a first step of the analysis, the relative treatment effect of mifamurtide in terms of EFS and its CI will be estimated using a Cox model with adjustment or stratification on stratification variables (chemotherapy regimen and risk group). 
The proportional hazards assumption will be evaluated using graphical methods and by extension of the Cox model including treatment-time interaction. As an exploratory analysis, the interaction between mifamurtide and the stratification factors (chemotherapy strata and risk group) will be investigated by adding an interaction term between treatment and each factor separately in the multivariable Cox model, in order to evaluate the heterogeneity of treatment effect across the different subgroups. Treatment effect will be estimated by subgroup (first (M)-API vs EI chemotherapy regimens; then the five risk groups) in the multivariable analysis, and reported in a forest plot. A similar approach will be used to evaluate the predictive value of some clinical and biological factors (exploratory analyses). The main analysis will be based on all patients included in the trial, regardless of protocol compliance (intention-to-treat analysis). Sensitivity analyses of EFS will be performed (1) on the per-protocol population, after exclusion of patients who have switched from one arm to the other as well as patients who could not undergo removal of all initially identified metastatic sites; (2) on the intention-to-treat population with adjustment or stratification on stratification variables and also other possible confounders. Absolute gain in EFS time will also be estimated using the restricted mean survival time difference ${ }^{1819}$ which remains valid if the PH assumption appears violated or questionable.

Similar statistical analyses will be used for the OS analysis. No interim analysis of efficacy is planned.

As some historical information is available (table 1), the usual hypothesis-driven approach (frequentist approach) will be completed with a Bayesian analysis incorporating the aggregate treatment effect estimated from a fixed-effect meta-analysis from the previous INT-0133 trial (in patients with localised osteosarcoma ${ }^{14}$; and metastatic disease $\left.^{15}\right)$ leading to an overall $\mathrm{HR}=0.786(\mathrm{SE}=0.110)$. We

Table 1 Sources of historical information

\begin{tabular}{|c|c|}
\hline \multicolumn{2}{|l|}{ Treatment effect; HR (95\% CI) } \\
\hline $\begin{array}{l}\text { INT-0133 } \text { trial }^{14} \\
(\mathrm{n}=662)\end{array}$ & $0.80(0.62$ to 1.00$)$ \\
\hline $\begin{array}{l}\text { INT-0133 trial } \\
(\mathrm{n}=91)\end{array}$ & $0.72(0.42$ to 1.20$)$ \\
\hline $\begin{array}{l}\text { Overall HR estimated from } \\
\text { the fixed-effect meta-analysis }\end{array}$ & $\begin{array}{l}0.786(\mathrm{SE}=0.110) \text { (no } \\
\text { heterogeneity across trial) }\end{array}$ \\
\hline \multicolumn{2}{|c|}{ Individual control arm (See figure 2) } \\
\hline \multicolumn{2}{|c|}{$\begin{array}{l}\text { Sarcome-09/OS2006 subgroup matching the following } \\
\text { criteria: } \\
\text { Patients <31 years old } \\
\text { Metastatic disease at diagnosis or non-responders to } \\
\text { chemotherapy } \\
\text { No event during the preoperative chemotherapy } \\
\text { Patients with surgery } \\
\text { Chemotherapy with methotrexate } \\
\text { Included in the control arm of the OS } 2006 \text { randomised tria } \\
\text { (256 patients, } 116 \text { events) }\end{array}$} \\
\hline
\end{tabular}

also plan to incorporate individual historical data, from Sarcome-09/OS2006 subgroup of patients who fulfilled the planned Sarcome-13/OS2016 eligibility criteria, on the control arm of the current trial (See figure 2).

We will use the approach proposed by Brard et al (BMC Medical Research Methodology-BMRM, in press) combining a mixture prior ${ }^{20}$ to incorporate the aggregate treatment effect from the INT-0133 trial, and a power prior $^{21}$ to incorporate the individual historical data from Sarcome-09/OS2006 subgroup of patients. The weights allocated to historical data ( $\omega$ and $\alpha_{0}$ for historical aggregate treatment effect and individual control data, respectively) have been calibrated based on a simulation study (see online supplementary materials). The trial will be considered successful if the posterior probability of $(\mathrm{HR}<1)$ is $\geq 90 \%$ (prespecified decision rule). Considering the chosen set of parameters resulting of the simulation study ( $\omega=0.1$ and $\alpha_{0}=0.3$, the power is increased from $80 \%$ (frequentist approach) to $98 \%$ (Bayesian approach) if the true treatment effect is $\mathrm{HR}=0.55$. It increases from $34 \%$ to $65 \%$ if the true treatment effect is consistent with the published INT-0133 data ( $\mathrm{HR}=0.786)$ (table 2). The posterior distribution of the HR will be described including the probability of $\mathrm{HR}<1$ or HR below different thresholds. Given the higher probability of positive conclusion of the planned Bayesian approach compared with the frequentist approach, it is possible the Bayesian success criteria will be met while the frequentist analysis is non-significant. In this case, we will claim a positive outcome for the trial based on the results of the Bayesian analysis. The 'power' of the proposed Bayesian approach is the probability of a positive conclusion considering the prespecified decision rule.

At the end of the trial, we may consider incorporating data from other relevant trials evaluating mifamurtide, which are currently in progress. A non-informative prior will also be considered to describe the distribution of the treatment effect when considering current trial data only. The Bayesian analysis will allow the description of the probability distribution of the treatment effect, such as the probability that HR is lower than various thresholds, not only $\mathrm{HR}<1$. The statistical analytical plan will be amended before data base lock to prespecify all sources of historical data.

For each type of $\mathrm{AE}$, the worst grade observed across the safety observational period ( 4 weeks after last treatment administration, and up to 24 weeks after last chemotherapy for control arm), will be tabulated by treatment $\mathrm{arm}$, and the percentages of severe $\mathrm{AE}$ (grade $\geq 4$ haematological $\mathrm{AE}$ and grade $\geq 3$ extrahaematological $\mathrm{AE}$ ) will be provided. A butterfly plot will be used to illustrate the difference in proportion of patients experiencing $\mathrm{AE}$ and severe $\mathrm{AE}$ between treatment groups. Relative risks of severe $\mathrm{AE}$ associated with mifamurtide will be estimated.

The Standard Protocol Items: Recommendations for Interventional Trials reporting guidelines have been used to report this trial protocol. ${ }^{22}$ 


\section{High-Risk osteosarcomas}

- Metastasis at diagnosis

- Or Localised disease and poor histological response

\section{Sarcoma 13}

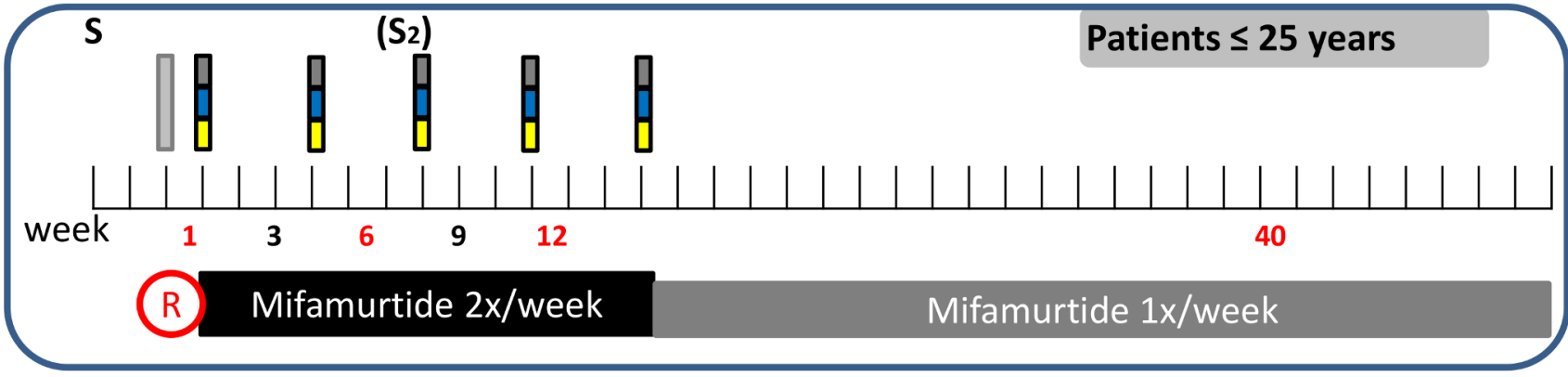

S

(S2)

Patients 26-50 years
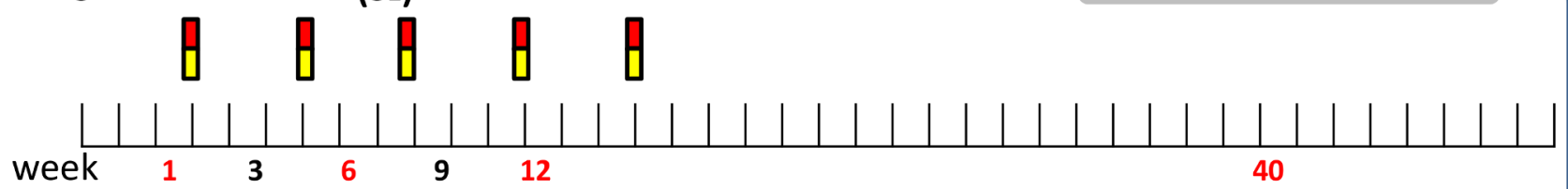

Mifamurtide $2 x /$ week

Mifamurtide 1x/week

MTX $12 \mathrm{~g} / \mathrm{m} 2 \times 1 \mathrm{~d}$
A : Adriamycine $60 \mathrm{mg} / \mathrm{m}^{2} \times 1 \mathrm{~d}$

$P$ : Cisplatinum $100 \mathrm{mg} / \mathrm{m}^{2} \times 1 \mathrm{~d}$

$\mathrm{I}$ : Ifosfamide $3 \mathrm{~g} / \mathrm{m}^{2} \times 2 \mathrm{~d}$
E : Etoposide $75 \mathrm{mg} / \mathrm{m}^{2} \times 4 \mathrm{~d}$

I : Ifosfamide $3 \mathrm{~g} / \mathrm{m}^{2} \times 4 \mathrm{~d}$

Figure 2 Individual historical data, from Sarcome-09/OS2006 subgroup of patients who fulfilled the planned Sarcome-13/ OS2016 eligibility criteria, on the control arm of the current trial.

\section{ETHICS AND DISSEMINATION}

The study is approved by the "Comite de Protection des Personnes Ile de France I" (CPP) and authorised by the National Agency for Medicines and Health Products

Table 2 Comparison of power and alpha error of the Sarcome-13/OS2016 trial between the frequentist approach and the Bayesian approach with $\omega=0.1$ and $\alpha_{0}=0.3$ for different 'true treatment effect'

\begin{tabular}{lll}
\hline & $\begin{array}{l}\text { Frequentist } \\
\text { approach, \% }\end{array}$ & $\begin{array}{l}\text { Bayesian } \\
\text { approach, \% }\end{array}$ \\
\hline $\begin{array}{l}\text { Scenario regarding the true } \\
\text { treatment effect }\end{array}$ & Power & \\
$\begin{array}{l}\text { HR=0.55 (anticipated effect } \\
\text { scenario) }\end{array}$ & 80 & 98 \\
$\begin{array}{l}\text { HR=0.786 (historical effect } \\
\text { scenario) }\end{array}$ & 34 & 65 \\
$\begin{array}{l}\text { HR=0.886 (disappointing } \\
\text { effect scenario) }\end{array}$ & 20 & 42 \\
\hline \begin{tabular}{l} 
HR=1 (null effect scenario) \\
\hline
\end{tabular} & 10 & 21 \\
\hline
\end{tabular}

Safety (ANSM) that reviewed the trial protocol, patient information sheets, informed consent forms, and other trial-related documents. If changes with substantial modifications occur, they will have to be submitted to the Agency for Medicines and Health Products Safety and to the Comite de Protection des Personnes Ile de France I by the sponsor. Data recorded during this trial are subject to a computerised treatment in compliance with the French law. The collection of biological samples has been notified to the French Ministry of Research. An independent data monitoring committee (IDMC), with expertise and experience in the pathology (two clinicians and one statistician), and without direct involvement in the conduct of the trial, will be set up. The IDMC will meet every 12 months and may recommend the early termination of the trial if an unacceptable toxicity occurred, or if the available data from the trial are sufficiently convincing to influence the therapeutic practices of the majority of clinicians. Investigators will make available to the authorised persons the documents and the patients' individual data that are essential to monitor the trial on an ongoing basis, to perform quality control and audit of this research in accordance with national regulatory requirements. 


\section{Patient and public involvement}

The protocol was designed and developed by paediatricians and medical oncologists who work in the InterSARC network that includes patients and parents, such as the 'Info Sarcomes' organisation. Patients were not directly involved in the design of the study; however, the protocol was discussed and approved by 'Info Sarcomes' organisation. The protocol has also been examined by the Patient Committee of the National League against Cancer who assessed the burden of the proposed intervention. Patients will be involved in recruitment to the study as the trial will be advertised on various websites (Info Sarcomes, Unicancer, hospitals' websites) promoting clinical studies to patients. Study results will be made available to study participants by being posted on ClinicalTrials.gov website.

\section{Dissemination}

The trial results, even if they are inconclusive, as well as biological ancillary studies, will be presented at appropriate international congresses and published in international peer review journals.

\section{Trial financing}

This study is sponsored by UNICANCER. It is mainly funded by the French Ministry of Health through the Hospital Clinical Research Program (PHRC-K-2016-130 grant). The study drug will be provided by Takeda to the sponsor.

\section{DISCUSSION}

This trial is a randomised phase II trial designed to detect an EFS benefit due to mifamurtide combined with postoperative chemotherapy compared with postoperative chemotherapy alone in patients aged $>2$ and $\leq 50$ with high-risk osteosarcoma. Considering the rare disease setting, a large phase III trial designed with the usual level of evidence would not have been feasible in a reasonable time frame at a national level, contrasting with the proposed design with a relaxed alpha error and a smaller sample size. The participation of other European centres has been discussed to increase the sample size. However, we had to cope with some major differences regarding the backbone chemotherapy, as most European countries use M-AP without ifosfamide whereas ifosfamide-based chemotherapy is used in all French centres. The possible interaction between mifamurtide and chemotherapy regimen (mainly ifosfamide based or not) could jeopardise the findings.

At the current stage of trial design, we cannot be sure that this trial will provide a definitive answer regarding the controversial role of mifamurtide in osteosarcoma, in particular, because of the relatively limited sample size (phase II trial). However, we think that it will contribute to address this issue in an appropriate way, as mifamurtide will be allocated by randomisation.

The proposed Bayesian analysis will help in combining evidence from the various trials to get the best treatment estimate and will increase the level of evidence on mifamurtide efficacy.

If the efficacy of mifamurtide is confirmed, this trial may contribute to allow the use of this promising drug, including in an extended population (extension to metastatic disease at diagnosis and older patients $>30$ to 50 years old).

\section{Author affiliations}

${ }^{1}$ CESP, INSERM, Faculté de médecine, Université Paris-Sud, Université Paris-Saclay, Villejuif, France

${ }^{2}$ Biostatistics and Epidemiology Department, Gustave Roussy, Villejuif, France ${ }^{3}$ Medical Oncology Department, Institut Curie, Paris, France

${ }^{4}$ UNICANCER, Paris, Île-de-France, France

${ }^{5}$ Department of Childhood and Adolescent Oncology, Gustave Roussy, Villejuif, France

${ }^{6}$ Medicaland Pharmaceutical Statistics Research Unit, Departmentof Mathematics and Statistics, FyldeCollege, Lancaster University, Lancaster, United Kingdom ${ }^{7}$ Statistical Methodology Department, Novartis Pharma AG, Basel, Switzerland ${ }^{8}$ Methodology and Biostatistic Unit, Centre Oscar Lambret, Lille, France

Acknowledgements We are grateful to all the physicians, surgeons, radiologists, pathologists, biologists who contributed to protocol writing, as well as to the whole UNICANCER sponsor team. We thank Estelle Lecointe, head of Info Sarcomes organisation, as well as other patient advisers for their review of the protocol and their support.

Contributors All authors designed the study; CB, GLT, M-CLD and NG contributed to the drafting of the manuscript. NG, SP-N, LB and JD contributed to the trial set-up. JD is responsible for data collection and for administrative support. M-CLD, $\mathrm{CB}, \mathrm{GLT}$ and LVH have proposed the statistical design and have performed the preliminary simulation study. CB, GLT and LVH will contribute to statistical analyses. M-CLD is responsible for data management and statistical analyses. All authors will contribute to data interpretation. All authors contributed to the revision of the manuscript and approved it for submission.

Funding The trial is supported by the French Ministry of Health (grant PHRC-K16130). The simulation study presented in online supplementary appendix of the paper has been funded by La Ligue contre le Cancer (PhD student program).

Competing interests None declared.

Patient consent for publication Not required.

Ethics approval The study is approved by the 'Comité de Protection des Personnes Ile de France l' (CPP) and authorised by the National Agency for Medicines and Health Products Safety (ANSM) that reviewed the trial protocol, patient information sheets, informed consent forms and other trial-related documents.

Provenance and peer review Not commissioned; externally peer reviewed.

Open access This is an open access article distributed in accordance with the Creative Commons Attribution Non Commercial (CC BY-NC 4.0) license, which permits others to distribute, remix, adapt, build upon this work non-commercially, and license their derivative works on different terms, provided the original work is properly cited, appropriate credit is given, any changes made indicated, and the use is non-commercial. See: http://creativecommons.org/licenses/by-nc/4.0/.

\section{REFERENCES}

1. Le Deley MC, Guinebretière JM, Gentet JC, et al. SFOP OS94: a randomised trial comparing preoperative high-dose methotrexate plus doxorubicin to high-dose methotrexate plus etoposide and ifosfamide in osteosarcoma patients. Eur J Cancer 2007;43:752-61.

2. Assi $H$, Missenard G, Terrier P, et al. Intensive induction chemotherapy without methotrexate in adult patients with localized osteosarcoma: results of the Institut Gustave-Roussy phase II trial. Curr Oncol 2010;17:23-31.

3. Piperno-Neumann S, Le Deley MC, Rédini F, et al. Zoledronate in combination with chemotherapy and surgery to treat osteosarcoma (OS2006): a randomised, multicentre, open-label, phase 3 trial. Lancet Oncol 2016;17:1070-80.

4. Isakoff MS, Bielack SS, Meltzer P, et al. Osteosarcoma: Current Treatment and a Collaborative Pathway to Success. J Clin Oncol 2015;33:3029-35. 
5. Asano T, McWatters A, An T, et al. Liposomal muramyl tripeptide up-regulates interleukin-1 alpha, interleukin-1 beta, tumor necrosis factor-alpha, interleukin-6 and interleukin-8 gene expression in human monocytes. J Pharmacol Exp Ther 1994;268:1032-9.

6. Kleinerman ES, Jia SF, Griffin J, et al. Phase II study of liposomal muramyl tripeptide in osteosarcoma: the cytokine cascade and monocyte activation following administration. J Clin Oncol 1992;10:1310-6.

7. Kleinerman ES, Murray JL, Snyder JS, et al. Activation of tumoricidal properties in monocytes from cancer patients following intravenous administration of liposomes containing muramyl tripeptide phosphatidylethanolamine. Cancer Res 1989;49:4665-70.

8. Nardin A, Lefebvre ML, Labroquère K, et al. Liposomal muramyl tripeptide phosphatidylethanolamine: Targeting and activating macrophages for adjuvant treatment of osteosarcoma. Curr Cancer Drug Targets 2006;6:123-33.

9. Pahl JH, Kwappenberg KM, Varypataki EM, et al. Macrophages inhibit human osteosarcoma cell growth after activation with the bacterial cell wall derivative liposomal muramyl tripeptide in combination with interferon- $\gamma$. J Exp Clin Cancer Res 2014;33:27.

10. Kurzman ID, MacEwen EG, Rosenthal RC, et al. Adjuvant therapy for osteosarcoma in dogs: results of randomized clinical trials using combined liposome-encapsulated muramyl tripeptide and cisplatin. Clin Cancer Res 1995;1:1595-601.

11. Vail DM, MacEwen EG, Kurzman ID, et al. Liposome-encapsulated muramyl tripeptide phosphatidylethanolamine adjuvant immunotherapy for splenic hemangiosarcoma in the dog: a randomized multiinstitutional clinical trial. Clin Cancer Res 1995;1:1165-70.

12. Kleinerman ES, Snyder JS, Jaffe N. Influence of chemotherapy administration on monocyte activation by liposomal muramyl tripeptide phosphatidylethanolamine in children with osteosarcoma. $J$ Clin Oncol 1991;9:259-67.

13. Meyers PA, Schwartz CL, Krailo M, et al. Osteosarcoma: a randomized, prospective trial of the addition of ifosfamide and/ or muramyl tripeptide to cisplatin, doxorubicin, and high-dose methotrexate. J Clin Oncol 2005;23:2004-11.

14. Meyers PA, Schwartz CL, Krailo MD, et al. Osteosarcoma: the addition of muramyl tripeptide to chemotherapy improves overall survival--a report from the Children's Oncology Group. J Clin Oncol 2008;26:633-8

15. Chou AJ, Kleinerman ES, Krailo MD, et al. Addition of muramyl tripeptide to chemotherapy for patients with newly diagnosed metastatic osteosarcoma: a report from the Children's Oncology Group. Cancer 2009;115:5339-48.

16. Anderson PM, Meyers P, Kleinerman E, et al. Mifamurtide in metastatic and recurrent osteosarcoma: a patient access study with pharmacokinetic, pharmacodynamic, and safety assessments. Pediatr Blood Cancer 2014;61:238-44.

17. Bielack S, Carrle D, Casali PG, et al. Osteosarcoma: ESMO Clinical Recommendations for diagnosis, treatment and follow-up. Annals of Oncology 2009;20(Supplement 4):iv137-iv139.

18. Irwin JO. The standard error of an estimate of expectation of life, with special reference to expectation of tumourless life in experiments with mice. J Hyg 1949;47:188-9.

19. Royston P, Parmar MK. The use of restricted mean survival time to estimate the treatment effect in randomized clinical trials when the proportional hazards assumption is in doubt. Stat Med 2011;30:2409-21.

20. Mutsvari T, Tytgat $D$, Walley R. Addressing potential prior-data conflict when using informative priors in proof-of-concept studies. Pharm Stat 2016;15:28-36.

21. Ibrahim JG, Chen M-H. Power prior distributions for regression models. Stat Sci 2000;15:46-60.

22. Chan AW, Tetzlaff JM, Altman DG, et al. SPIRIT 2013 statement: defining standard protocol items for clinical trials. Ann Intern Med 2013;158:200-7. 